\title{
Inhibition of IRE1 signaling affects the expression of genes encoded glucocorticoid receptor and some related factors and their hypoxic regulation in $\mathrm{U} 87$ glioma cells
}

\author{
${ }^{1,2}$ Minchenko DO, ${ }^{1}$ Riabovol OO, ${ }^{1}$ Tsymbal DO, ${ }^{1}$ Ratushna OO, ${ }^{1}$ Minchenko OH
}

${ }^{1}$ Department of Molecular Biology, Palladin Institute of Biochemistry, National Academy of Sciences of Ukraine, Kyiv, Ukraine; ${ }^{2}$ Department of Pediatrics, National Bohomolets Medical University, Kyiv, Ukraine

E-mail: ominchenko@yahoo.com

Objective. The aim of the present investigation was to examine the effect of inhibition of endoplasmic reticulum stress signaling, mediated by IRE1 (inositol requiring enzyme 1), which is a central mediator of the unfolded protein response on the expression of genes encoding glucocorticoid receptor (NR3C1) and some related proteins (SGK1, SGK3, NCOA1, NCOA2, ARHGAP35, NNT) and their hypoxic regulation in U87 glioma cells for evaluation of their possible significance in the control of the glioma growth.

Methods. The expression of NR3C1,SGK1,SGK3, NCOA1, NCOA2, ARHGAP35, and NNT genes in U87 glioma cells, transfected by empty vector pcDNA3.1 (control) and cells without IRE1 signaling enzyme function (transfected by dnIRE1) upon hypoxia, was studied by quantitative polymerase chain reaction.

Results. Inhibition of IRE1 signaling enzyme function up-regulates the expression of $\mathrm{NR} 3 \mathrm{Cl}$, SGK1, NCOA1, NCOA2, ARHGAP35, and NNT genes in U87 glioma cells in comparison with the control glioma cells, with more significant changes for NR3C1, SGK1, and NNT genes. At the same time, the expression of $S G K 3$ gene is strongly down-regulated in glioma cells upon inhibition of IRE1. We have also shown that hypoxia increases the expression of NR3C1, SGK1, NCOA2, ARHGAP35, and NNT genes but decreases SGK3 and NCOA1 genes expression in control glioma cells. Moreover, the inhibition of both enzymatic activities (kinase and endoribonuclease) of IRE1 in U87 glioma cells enhances the effect of hypoxia on the expression of SGK1, SGK3, and NNT genes, but decreases the sensitivity of $\mathrm{NR} 3 \mathrm{C} 1$ gene to hypoxic condition. Furthermore, the expression of NCOA1 gene is resistant to hypoxia in control glioma cells, but NCOA2 and ARHGAP35 genes are resistant to this condition in glioma cells without functional activity of IRE1 signaling enzyme.

Conclusions. Results of this investigation demonstrate that inhibition of IRE1 signaling enzyme function affects the expression of NR3C1, SGK1, SGK3, NCOA1, NCOA2, ARHGAP35, and NNT genes in U87 glioma cells in gene specific manner and that all these genes are regulated by hypoxia preferentially through IRE1 signaling pathway of the endoplasmic reticulum stress.

Key words: IRE1 inhibition, mRNA expression, NR3C1, SGK1, SGK3, NCOA2, ARHGAP35, NNT, hypoxia, U87 glioma cells 
Glucocorticoid receptor (nuclear receptor subfamily 3, group $\mathrm{C}$, member 1; NR3C1) plays an important role in the regulation of numerous metabolic and proliferative processes, including tumorigenesis and metabolic diseases, such as obesity and diabetes, preferentially through interaction with specific response elements in the target genes (Vonlanthen et al. 2014; de Guia et al. 2015; Kim et al. 2015; Pufall 2015; Thomas et al. 2015). Unlike other steroid hormone receptors, the glucocorticoid receptor is not considered to be an oncogene. Glucocorticoids work through $\mathrm{NR} 3 \mathrm{C} 1$ to arrest the growth and induce apoptosis in lymphoid tissue (Pufall 2015; Huang et al. 2016). Furthermore, transcriptional activity of NR3C1 correlates with the amount of phosphorylation at Ser-211, but its sumoylation reduces the transcription transactivation. Glucocorticoids can stimulate directly the mitochondrial transcription through glucocorticoid receptors (Minchenko and Germanyuk 1984; Minchenko 1988; Minchenko and Tronjko 1988; Psarra and Sekeris 2011). Moreover, glucocorticoid receptors can also signal by binding to other transcription factors and modulate the transcriptional regulation of target genes (Khan et al. 2011; Li et al. 2012; Dasgupta et al. 2015).

Therefore, glucocorticoid receptor DNA binding factor 1 (GRF-1), also known as Rho GTPase Activating Protein 35 (ARHGAP35), is a repressor of glucocorticoid receptor transcription and represses the transcription of this receptor by binding to the cis-acting regulatory sequence (Notsuda et al. 2013; Organ et al. 2014; Zhao et al. 2014). Nuclear receptor coactivator 2 (NCOA2), also known as a glucocorticoid receptor-interacting protein-1 (GRIP1), is a critical regulator of glucose metabolism, involved in the positive regulation of the transcriptional activity of the glucocorticoid receptor NR3C1. However, it also regulates other steroid receptors as well as thyroid, retinoid, and vitamin D receptors (Fenne et al. 2013; Liu et al. 2013; Dasgupta et al. 2015).

Glucocorticoids are responsible for the regulation of SGK1 and SGK3 (serum/glucocorticoid regulated kinases 1 and 3), which also are induced by a very large spectrum of stimuli distinct from glucocorticoids and serum (Srivastava et al. 2014; Hausmann et al. 2015; Liu et al. 2015; Qin et al. 2015; Xiaobo et al. 2016). Nicotinamide nucleotide transhydrogenase (NNT) is an integral protein of the inner mitochondrial membrane and functions as a proton pump across the membrane. At the same time, mutations in NNT gene cause familial glucocorticoid deficiency (Meimaridou et al. 2012). NNT mRNA expression is significantly higher in visceral fat of obese patients and correlates with body weight and fasting plasma insulin (Heiker et al. 2013). Moreover, in the failing heart, a partial loss of NNT activity adversely impacts NADPH-dependent enzymes and the capacity to maintain membrane potential, thus contributing to a decline in bioenergetic capacity, redox regulation and antioxidant defense (Sheeran et al. 2010).

Regulation of different metabolic processes by glucocorticoids is preferentially stress dependent, but involving the endoplasmic reticulum (ER) stress signaling has not been explored yet. The ER stress is mediated by three sensor and signaling pathways, but inositol requiring enzyme 1 (IRE1/ERN1) is a central mediator of the unfolded protein response and an important component of tumor growth, because its inhibition leads to a suppression of glioma growth through down-regulation of the angiogenesis and proliferation processes (Drogat et al. 2007; Auf et al. 2010, 2013). This stress is recognized as an important determinant of cancer, obesity, and type 2 diabetes and contributes to the expression profile of many regulatory genes resulting in proliferation, apoptosis, angiogenesis, and insulin resistance (Minchenko et al. 2013a,b; Clarke et al. 2014; Han and Kaufman 2014; Lee and Ozcan 2014; Manie et al. 2014; Dejeans et al. 2015; Minchenko et al. 2015a). The IRElenzyme is responsible for the degradation of a specific subset of mRNA and alternative splicing of the XBP1 (X-box binding protein 1) transcription factor mRNA for control of the expression of unfolded protein response-specific genes (Acosta-Alvear et al. 2007; Aragon et al. 2009; Auf et al. 2010; Dejeans et al. 2012; Han et al. 2013; Pluquet et al. 2013; Maurel et al. 2014, 2015).

Moreover, the ER has an essential position as a signal integrator because of the signaling pathways, elicited by ER stress sensors, have connections with the metabolic pathways and with other plasma membrane receptor signaling networks (Bravo et al. 2013; Cao and Kaufman 2013; Han et al. 2013; Clarke et al. 2014; Manie et al. 2014; Chevet et al. 2015). The ER stress response-signaling pathway, mediated by IRE1, is tightly associated with the hypoxia and linked to the neovascularization, tumor growth, and cell death processes as well as suppression of insulin receptor signaling through activation of c-Jun N-terminal kinase (Langlais et al. 2011; Lenihan and Taylor 2013; Clarke et al. 2014; Minchenko et al. 2014).

The main goal of this work was to study: 1) the effect of signaling enzyme IRE1 inhibition on the expression level of genes encoding the NR3C1, SGK1, SGK3, NCOA1, NCOA2, ARHGAP35, and NNT in U87 glioma cells and 2) the regulation of these gene 
expressions by hypoxia for evaluation of its possible significance in the control of glioma growth through IRE1 mediated ER stress signaling.

\section{Materials and Methods}

Cell Lines and Culture Conditions. The glioma cell line U87 was obtained from ATCC (U.S.A.) and grown in high glucose $(4.5 \mathrm{~g} / \mathrm{l})$ Dulbecco's modified Eagle's minimum essential medium (Gibco, Invitrogen, Carlsbad, CA, U.S.A.), supplemented with glutamine $(2 \mathrm{mM}), 10 \%$ fetal bovine serum (EquitechBio, Inc., U.S.A.), penicillin (100 units/ml; Gibco) and streptomycin $(0.1 \mathrm{mg} / \mathrm{ml} ; \mathrm{Gibco})$ at $37^{\circ} \mathrm{C}$ in a $5 \% \mathrm{CO}_{2}$ incubator. In this work, we used two sublines of this glioma cells. One subline was obtained by selection of stable transfected clones with over expression of vector pcDNA3.1, which was used for creation of dnIRE1. This untreated subline of glioma cells (control glioma cells) was used as control 1 in the study of the effect of hypoxia on the expression level of glucocorticoid receptor and related genes. Second subline was obtained by selection of stable transfected clone with over expression of IRE1 dominant/negative construct (dnIRE1) and has suppressed both protein kinase and endoribonuclease activities of this signaling enzyme (Auf et al. 2010). The expression level of studied genes in these cells was compared with cells, transfected by vector (control 1), but this subline was also used as control 2 for investigation the effect of hypoxia on gene expressions under blockade of both enzymatic activities of IRE1. The efficiency of IRE1 suppression in this glioma cell subline was estimated previously (Auf et al. 2010, 2013; Minchenko et al. 2013b) by determining the expression level of the XBP1 alternative splice variant, a key transcription factor in the IRE1 signaling, and the level of the phosphorylated isoform IRE1 using cells treated by tunicamycin $(0.01 \mathrm{mg} / \mathrm{ml}$ during $2 \mathrm{~h}$ ) for induction of ER stress. Both sublines of glioma cells, used in this study, are grown with the addition of geneticin (G418) while these cells carrying empty vector pcDNA3.1 or dnIRE1 construct.

Hypoxic condition was created in special incubator with $3 \%$ oxygen and $5 \%$ carbon dioxide levels. The culture plates with complete DMEM were exposed to these conditions for $16 \mathrm{~h}$.

RNA isolation. Total RNA was extracted from glioma cells using the Trizol reagent (Invitrogen, Carlsbad, CA, U.S.A.). RNA pellets was washed with $75 \%$ ethanol and dissolved in nuclease-free water. For additional purification RNA samples were reprecipitated with $95 \%$ ethanol and re-dissolved in nuclease-free water. RNA concentration and spectral characteristics were measured using a NanoDrop Spectrophotometer ND1000 (PEQLAB, Biotechnologie $\mathrm{GmbH}$ ).

Reverse transcription and quantitative PCR analysis. The expression levels of glucocorticoid receptor (nuclear receptor subfamily 3, group C, member 1; NR3C1), serum/glucocorticoid regulated kinase 1 (SGK1), serum/glucocorticoid regulated kinase family, member 3 (SGK3),nuclear receptor corepressor 1 (NCOA1), nuclear receptor coactivator 2 (NCOA2), also known as glucocorticoid receptor-interacting protein-1, Rho GTPase Activating Protein 35 (ARHGAP35), also known as glucocorticoid receptor DNA binding factor 1 (GRLF1), and nicotinamide nucleotide transhydrogenase (NNT) mRNAs as well as ACTB mRNA were measured in control U87 glioma cells and cells with a deficiency of IRE1 by quantitative polymerase chain reaction in real-time using qPCR „RotorGene RG-3000” (Corbett Research, Germany) and Applied Biosystems 7500 (Applied Biosystems, U.S.A.) using SYBRGreen Mix (ABgene, Thermo Fisher Scientific, Epsom, Surrey, UK). QuaniTect Reverse Transcription Kit (QIAGEN, Hilden, Germany) was used for cDNA synthesis as described previously (Minchenko et al. 2013b). Polymerase chain reaction was performed in triplicate.

For amplification of NR3C1 cDNA, we used forward (5'-tttccetcctgctccttctg-3' and reverse (5'-tcacatctcccctctcctga- $3^{\prime}$ ) primers. The nucleotide sequences of these primers correspond to sequences 382-401 and 571-552 of human NR3C1 cDNA (GenBank accession number NM_000176). The amplification of SGK1 cDNA for real time RCR analysis was performed using two oligonucleotides primers: forward - 5'-gcagaaggacaggacaaagc-3' and reverse - 5'-tcggtaaactcggggtcaaa-3'. The nucleotide sequences of these primers correspond to sequences 1085-1104 and 1261-1242 of human SGK1 cDNA (GenBank accession number NM_005627). For amplification of SGK3 cDNA we used forward (5'-attcccagctccgatgaaca- $3^{\prime}$ and reverse (5'-tcgtttagtcctgctcgtct- $3^{\prime}$ ) primers. The nucleotide sequences of these primers correspond to sequences 312-331 and 547-528 of human SGK3 cDNA (GenBank accession number NM_013257). The amplification of ARHGAP35 cDNA for real time RCR analysis was performed using two oligonucleotides primers: forward - 5'-ggcaacctgggagagtaact-3' and reverse 5 '-agtctttctctgccaggtcc- 3 '. The nucleotide sequences of these primers correspond to sequences 3717-3736 and 3934-3915 of human ARHGAP35 cDNA (GenBank accession number NM_004491). For amplification of NCOA1 cDNA we used two oligonucleotides 
primers: forward - 5'-aatgatcccgcactgagaca- $3^{\prime}$ and reverse - 5'-ggcttttgagttcccagtgg-3'. The nucleotide sequences of these primers correspond to sequences 4327-4346 and 4517-4498 of human NCOA1 cDNA (GenBank accession number NM_003743). The amplification of NCOA2 cDNA for real time RCR analysis was performed using two oligonucleotides primers: forward - 5'-gcccagagacagagggaaat-3' and reverse - 5'-cgagggttgctcatagttgc-3'. The nucleotide sequences of these primers correspond to sequences 3868-3887 and 4031-4012 of human NCOA2 cDNA (GenBank accession number NM_006540). For amplification of NNT cDNA we used two oligonucleotides primers: forward - 5'-gtctcctgaaatctgcccct-3' and reverse - 5'-cagcacagtgataacgacgg-3'. The nucleotide sequences of these primers correspond to sequences $2549-2568$ and $2769-2750$ of human NNT cDNA (GenBank accession number NM 012343). The amplification of ACTB (beta-actin) cDNA for

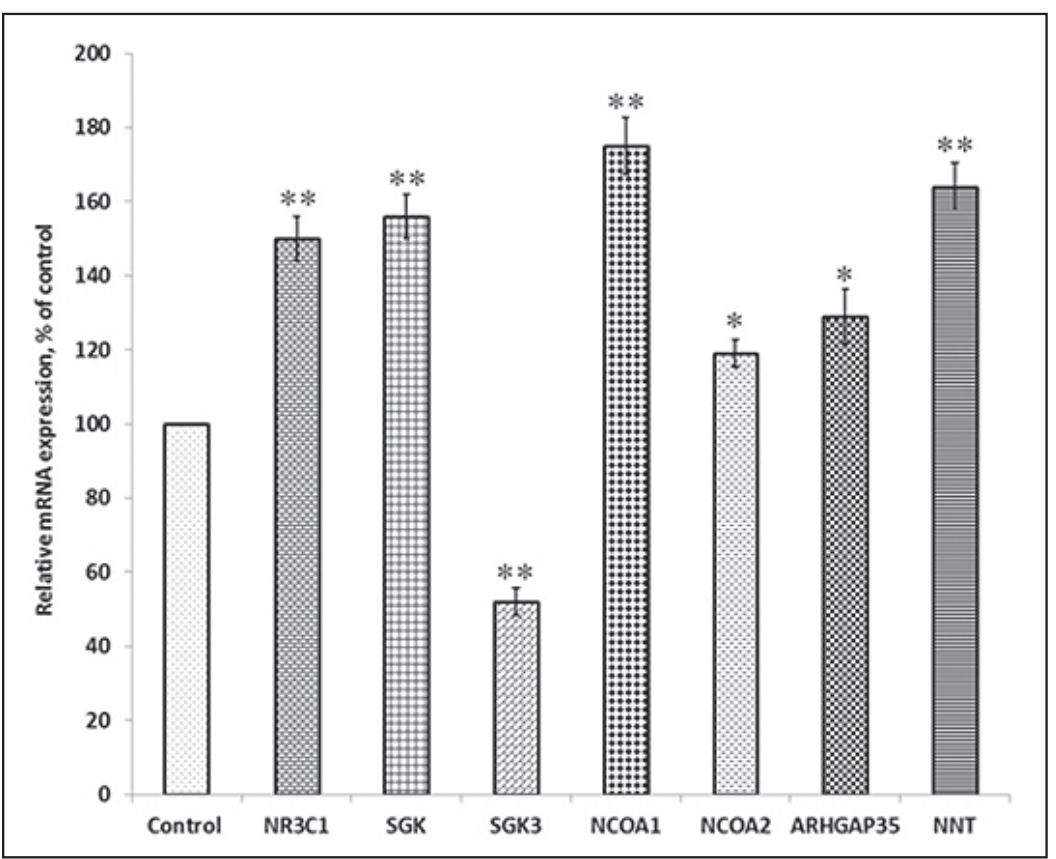

Figure 1. Inhibition of IRE1 by dnIRE1 affects the expression level of genes encoded glucocorticoid receptor (nuclear receptor subfamily 3, group C, member 1; NR3C1), serum/glucocorticoid regulated kinase 1 (SGK1), serum/glucocorticoid regulated kinase family, member 3 (SGK3), nuclear receptor corepressor 1 (NCOA1), nuclear receptor coactivator 2 (NCOA2), also known as glucocorticoid receptor-interacting protein-1, Rho GTPase Activating Protein 35 (ARHGAP35), also known as glucocorticoid receptor DNA binding factor 1 (GRLF1), and nicotinamide nucleotide transhydrogenase (NNT) in U87 glioma cells measured by qPCR. Values of these mRNA expressions were normalized to beta-actin mRNA level and represented as percent for control (cells transfected be empty vector, $100 \%$ ); $n=4 ;{ }^{*} \mathrm{p}<0.05$ and ${ }^{*} \mathrm{p}<0.01$ vs. control. real time RCR analysis was performed using forward - 5'-ggacttcgagcaagagatgg-3' and reverse - 5'-agcactgtgttggcgtacag- $3^{\prime}$ primers. These primers nucleotide equences correspond to 747-766 and 980-961 of received from Sigma-Aldrich (St. Louis,

Quantitative PCR analysis was performed using a special computer program "Differential expression calculator". The values of NR3C1, SGK1, SGK3, NCOA1, NCOA2, ARHGAP35, and NNT gene expressions were normalized to the expression of beta-actin CTB) mRNA and represent as percent of control ). All values are expressed as mean \pm SEM from experiments. Statistical analysis was performed as described previously (Bochkov et al 2006). The amplified DNA fragments were also analyzed on a $2 \%$ agarose gel and that visualized by $\mathrm{SYBR}^{*} \mathrm{Safe}$ DNA Gel Stain (Life Technologies, Carlsbad, CA, U.S.A.).

\section{Results}

Inhibition ofIRE1 affects the expression of NR3C1, SGK1, SGK3, NCOA1, NCOA2, ARHGAP35, and NNT genes in U87 glioma cells. To investigate a possible role of ER stress signaling, mediated by IRE1 bifunctional enzyme, in the expression level of mRNA for glucocorticoid receptor and related proteins, we studied the effect of IRE1 inhibition by dnIRE1 on these genes expression in U87 glioma cells. As shown in Figure 1, the expression level of NR3C1 mRNA is significantly upregulated $(+50 \%)$ in U87 glioma cells by inhibition of IRE1 signaling enzyme in comparison with the control cells. The expression level of SGK1 mRNA in glioma cells without IRE1 signaling enzyme function is also up-regulated $(+56 \%)$ in comparison with the control glioma cells. At the same time, inhibition of IRE1 by dnIRE1 strongly down-regulated 


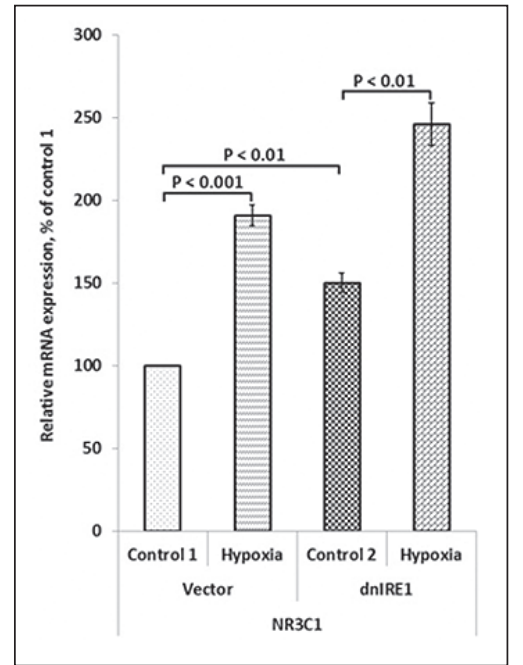

Figure 2. Effect of hypoxia on the expression level of glucocorticoid receptor (nuclear receptor subfamily 3, group $\mathrm{C}$, member 1; NR3C1) mRNA in control U87 glioma cells (Vector) and cells with a blockade of the IRE1 by dnIRE1 measured by qPCR. Values of NR3C1 mRNA expressions were normalized to betaactin mRNA level and represented as percent for control $1(100 \%) ; n=4$.

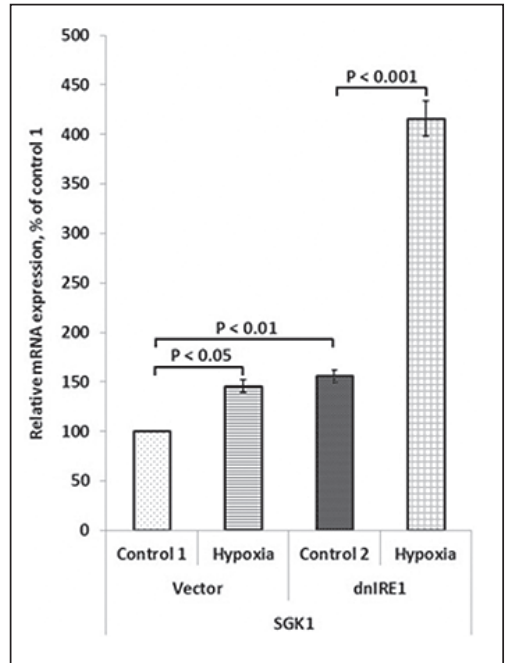

Figure 3. Effect of hypoxia on the expression level of serum/glucocorticoid regulated kinase 1 (SGK1) mRNA in control U87 glioma cells (Vector) and cells with a blockade of the IRE1 by dnIRE1 measured by qPCR. Values of SGK1 mRNA expressions were normalized to betaactin mRNA level and represent as percent for control $1(100 \%) ; n=4$.

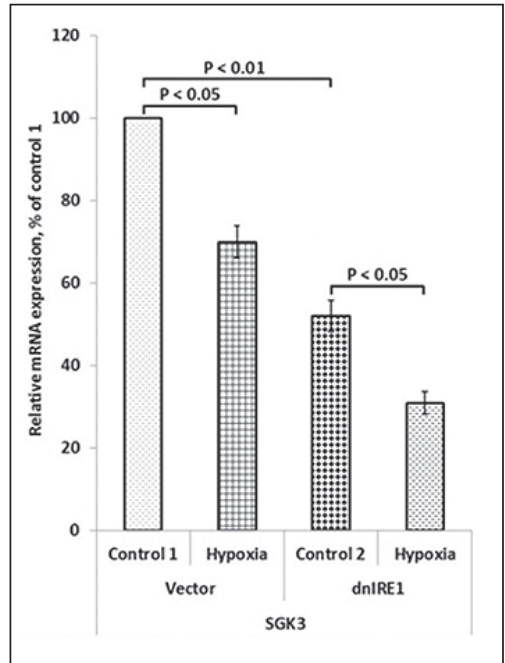

Figure 4. Effect of hypoxia on the expression level of serum/glucocorticoid regulated kinase family, member 3 (SGK3) mRNA in control U87 glioma cells (Vector) and cells with a blockade of the IRE1 by dnIRE1 measured by qPCR. Values of SGK3 mRNA expressions were normalized to beta-actin mRNA level and represented as percent for control $1(100 \%) ; n=4$. the expression level of SGK3mRNA (-48\%) in glioma cells in comparison with the control ones (Figure 1). Furthermore, we investigated the expression of genes encoded nuclear receptor coactivator/corepressor 1 (NCOA1) and nuclear receptor coactivator 2 (NCOA2), also known as glucocorticoid receptor-interacting protein-1, as well as glucocorticoid receptor DNA binding factor 1 (GRLF1), also known as Rho GTPase Activating Protein 35 (ARHGAP35), and nicotinamide nucleotide transhydrogenase (NNT) in relation to the inhibition of IRE1 signaling enzyme function. As also shown in Figure 1, the expression level of NCOA1 and NNT mRNAs is strongly increased ( $+75 \%$ and $+64 \%$, correspondingly) in glioma cells with suppressed function of IRE1 signaling enzyme. Smaller but statistically significant induction was shown for ARHGAP35 (+29\%) and NCOA2 genes $(+19 \%)$. The inhibition of ER stress signaling, mediated by IRE1 enzyme, affected the expression level of glucocorticoid receptor and a subset of related factors in gene-specific manner.

Effect of hypoxia on the expression of NR3C1, SGK1, SGK3, NCOA1, NCOA2, ARHGAP35, and NNT genes in control glioma cells and cells with- out IRE1 enzyme function. Furthermore, we investigated the effect of hypoxia on the expression of NR3C1, SGK1, SGK3, NCOA1, NCOA2, ARHGAP35, and NNT genes in glioma cells in relation to inhibition of IRE1 signaling enzyme function. We found that expression level of $\mathrm{NR} 3 \mathrm{C} 1$ gene is strongly upregulated by hypoxia in control glioma cells $(+91 \%$ in comparison with control 1) and that inhibition of IRE1 signaling enzyme function decreases the effect of hypoxia on this gene expression (+64\% in comparison with control 2) (Figure 2). As shown in Figure 3 , hypoxia also significantly enhances the expression level of SGK1 gene in the control glioma cells in comparison with the control $1(+46 \%)$. At the same time, inhibition of IRE1 signaling enzyme strongly increases the sensitivity of SGK1 gene expression to hypoxia. Thus, in the glioma cells without IRE1 signaling enzyme function the expression level of this gene is up-regulated 2.7 times in comparison with corresponding control (control 2; Figure 3). Investigation of $S G K 3$ gene expression had shown that hypoxia down-regulated its expression in both the control glioma cells and cells without IRE1 signaling enzyme function. Thus, as shown in Figure 4, the ex- 


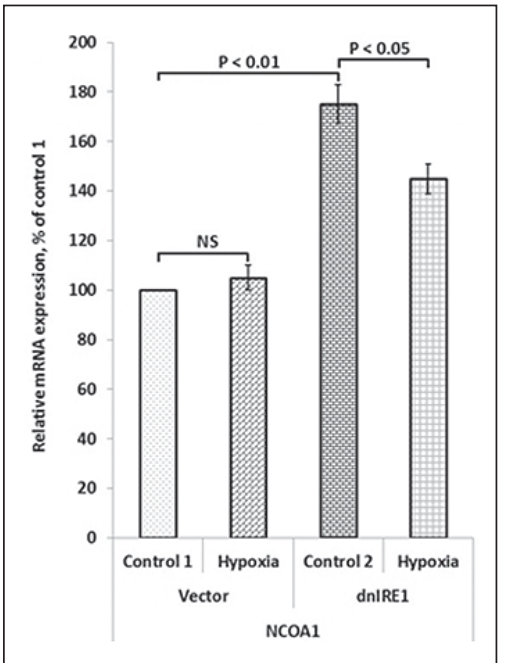

Figure 5. Effect of hypoxia on the expression level of nuclear receptor corepressor 1 (NCOA1) mRNA in control U87 glioma cells (Vector) and cells with a blockade of the IRE1 by dnIRE1 measured by qPCR. Values of NCOA1 mRNA expressions were normalized to beta-actin mRNA level and represented as percent for control $1(100 \%)$; $\mathrm{n}=4$.

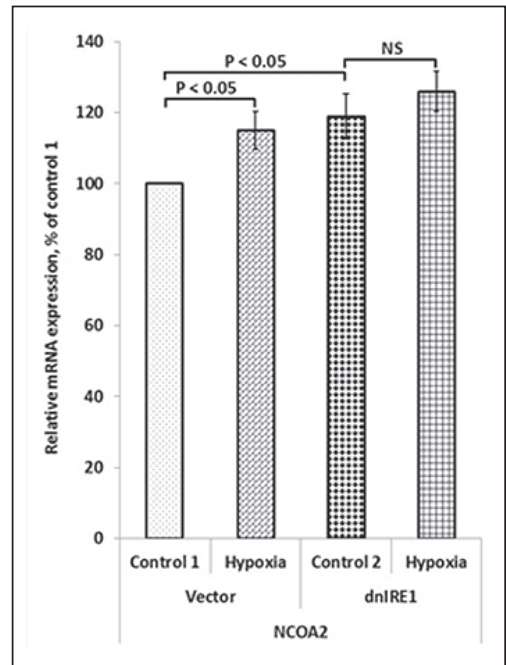

Figure 6. Effect of hypoxia on the expression level of nuclear receptor coactivator 2 (NCOA2), also known as glucocorticoid receptor-interacting protein-1,mRNA in control U87 glioma cells (Vector) and cells with a blockade of the IRE1 by dnIRE1 measured by qPCR. Values of NCOA2 mRNA expressions were normalized to beta-actin mRNA level and represented as percent for control 1 (100\%); $\mathrm{n}=4$.

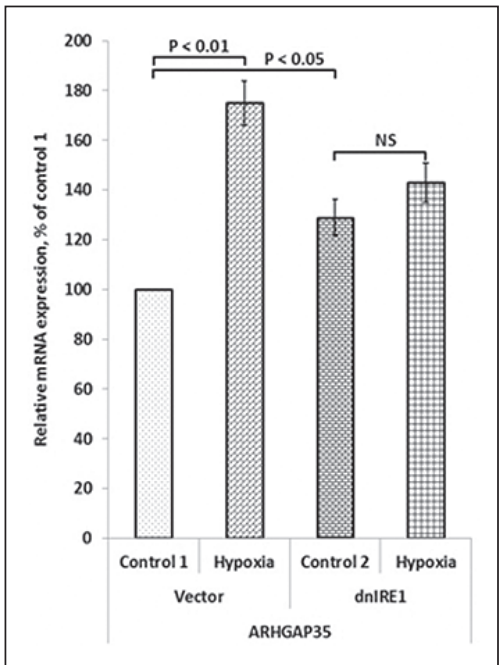

Figure 7. Effect of hypoxia on the expression level of Rho GTPase Activating Protein 35 (ARHGAP35), also known as glucocorticoid receptor DNA binding factor 1 (GRLF1), mRNA in control U87 glioma cells (Vector) and cells with a blockade of the IRE1 by dnIRE1 measured by qPCR. Values of ARHGAP35 mRNA expressions were normalized to beta-actin mRNA level and represented as percent for control 1 $(100 \%) ; \mathrm{n}=4$. pression level of $S G K 3$ gene is decreased by hypoxia $(-30 \%)$ in control glioma cells in comparison with control land inhibition of IRE1 slightly enhances the effect of hypoxia on this gene expression $(-40 \%)$ in comparison with control 2.

We also studied the hypoxic regulation of NCOA1 and NCOA2 genes expression in the glioma cells in relation to inhibition of IRE1 signaling enzyme function. We found that in control glioma cells, hypoxia is slightly but statistically significantly up-regulated the expression level of NCOA2 gene ( $+15 \%$ in comparison with control 1) and does not affect NCOA1 the gene expression (Figures 5 and 6 ). At the same time, inhibition of IRE1 signaling enzyme function eliminates hypoxic regulation of NCOA2 gene expression and introduces sensitivity to hypoxia the expression of NCOA1 gene (-17\% in comparison with control 2) in U87 glioma cells. Thus, effect of hypoxia on these two genes encoded nuclear receptor coactivator proteins is different but mediated through IRE1 signaling pathway.

Investigation of glucocorticoid receptor DNA binding factor 1 gene expression, also known as Rho
GTPase Activating Protein 35 (ARHGAP35), showed that hypoxia strongly up-regulated its expression (+75\% in comparison with control 1) and that inhibition of IRE1 enzyme function by dnIRE1 eliminated its hypoxic regulation (Figure 7). At the same time, effect of hypoxia on the expression level of NNT gene is significantly lower. As shown in Figure 8, hypoxia slightly but statistically significantly up-regulated the expression level of this gene in control glioma cells (+16\% in comparison with control 1), but inhibition of IRE1 signaling enzyme significantly enhanced the hypoxic regulation of NNT gene expression $(+42 \%$ in comparison with control 2).

Thus, the expression of genes, encoded by glucocorticoid receptor and a subset of related factors, is responsible for the endoplasmic reticulum stress signaling, mediated by IRE1 and hypoxia in glioma cells, in gene specific manner.

\section{Discussion}

In this work, we studied the expression of genes encoded glucocorticoid receptor and a subset of re- 


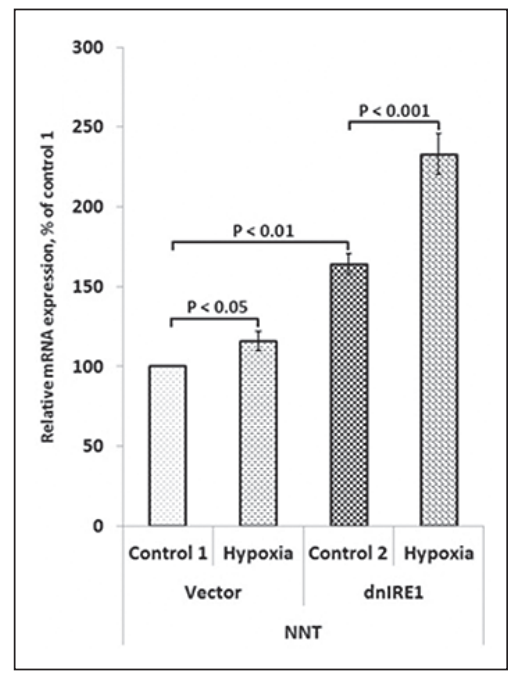

Figure 8. Effect of hypoxia on the expression level of nicotinamide nucleotide transhydrogenase (NNT) mRNA in control U87 glioma cells (Vector) and cells with a blockade of the IRE1 by dnIRE1 measured by qPCR. Values of NNT mRNA expressions were normalized to betaactin mRNA level and represented as percent for control 1 (100\%); $\mathrm{n}=4$.

lated factors, which regulate its functional activity and expression in relation to hypoxia and inhibition of IRE1, the major signaling pathway of the unfolded protein response, in U87 glioma cells. It is important for evaluation of possible significance of these genes in the control of glioma growth through ER stress signaling, mediated by IRE1 and hypoxia because stress signaling pathways is involved in numerous metabolic pathways and inhibition of the activity of IRE1 signaling enzyme in glioma cells had anti-tumor effects (Auf et al. 2010, 2013; Bravo et al. 2013; Minchenko et al. 2013b; Manie et al. 2014; Minchenko et al. 2014, 2015b).

Results of this study demonstrated that inhibition of IRE1 signaling enzyme function sig- nificantly increases the expression level of both $N R 3 C 1$ and most related genes, indicating their participation in IRElmediated network of the unfolded protein response. The changes in the glucocorticoid receptor and related factors expression in cells without IRE1 signaling enzyme function possible contribute in the suppression of glioma cell proliferation and tumor growth, because there is data that glucocorticoid receptor plays an important role in growth arrest and dexamethasonemediated killing of $\mathrm{B}$ lymphoma cells by induction of apoptosis ( $\mathrm{Pu}-$ fall 2015; Huang et al. 2016). It is possible that increased expression of GRF-1/ARHGAP35, NNT, NCOA1, and GRIP1/NCOA2 genes (Figure 1) contributes to the suppression of proliferation and glioma growth from glioma cells with IRE1 knockdown (Auf et al. 2010, 2013; Minchenko et al. 2015b). Thus, increased expression of nicotinamide nucleotide transhydrogenase should enhance glucocorticoid receptor function because mutations in NNT cause familial glucocorticoid deficiency (Meimaridou et al. 2012).

However, almost all studied glucocorticoid related factors, especially serum/glucocorticoid regulated kinases and nuclear receptor coactivator/corepressor factors, are multifunctional and their biological effects are dependent on ER stress signaling pathways and many other factors in cell specific manner (Khan et al. 2011; Li et al. 2012; Fenne et al. 2013; Liu et al. 2013; Chi et al. 2015; Dasgupta et al. 2015).Therefore, our results conform the data indicating that NR3C1,ARHGAP35, and NCOA2 have mainly anti-proliferative functions through interaction with different transcription co-regulators and signaling pathways of ER stress in cell specific manner (Pufall 2015; Kim et al. 2015; Huang et al. 2016). At the same time, we have shown that SGK3 gene expression is down-regulated by inhibition of IRE1. These results conform to data that inositol polyphosphate 4-phosphatase type II in melanoma functions as an oncogenic driver through activation of serum- and glucocorticoid-regulated kinase 3 (Chi et al. 2015).

Consequently, our results demonstrate that all of the genes studied are ER stress responsive playing an essential role in the control of glucocorticoid hormone action and cell proliferation, but functional significance and molecular mechanisms of induction or suppression of these genes expression upon inhibition of IRE1 are not clear and many details remain to be elucidated. Thus, the ER has an important position as a signal integrator in both normal and malignant cells as well as in the cells upon metabolic diseases because its signaling pathways is involved in numerous metabolic pathways (Bravo et al. 2013; Cao and Kaufman 2013; Minchenko et al. 2013b; Han and Kaufman 2014; Lee and Ozcan 2014; Manie et al. 2014; Pluquet et al. 2014; Chevet et al. 2015).

The investigation of hypoxic regulation of the expression of genes, encoded glucocorticoid receptor and related factors in respect to inhibition of IRE1 signaling, is very important for understanding of malignant tumor growth mechanisms, because low oxygen has essential role in the control of proliferation and tumor progression (Lenihan and Taylor 2013; Minchenko et al. 2013a, 2015a). We showed that hypoxia leads to up-regulation of the expression of NR3C1, SGK1, NCOA2, ARHGAP35, and NNT genes in control glioma cells and that inhibition of IRE1 signaling enzyme function modifies hypoxic regulation in all studied genes. Thus, the effect of hypoxia on the expression level of $N R 3 C 1$ gene is decreased by inhibition of IRE1 signaling enzyme in glio- 
ma cells, but significantly increased in the expression of SGK1 and NNT genes. Moreover, IRE1 knockdown eliminates effect of hypoxia on the expression level of NCOA2 and ARHGAP35 genes. Thus, inhibition of IRE1 modifies the sensitivity of some studied genes expression to hypoxia and induces negative effect of IRE1 inhibition on NCOA1 gene expression as well as reduces effect of hypoxia on the expression of NR3C1 gene in glioma cells. These changes in the expression of genes encoded glucocorticoid receptor and related factors in control glioma cells upon hypoxia are similar to that in cells without IRE1 signaling enzyme function. It is not clear yet why the hypoxia up-regulates the expression of genes encoded glucocorticoid receptor and some related factors, which expression is increased by IRE1 inhibition and is correlated with anti-proliferative effect.

This study provides unique insights into the molecular mechanisms, regulating the expression of genes encoding glucocorticoid receptor and a subset of related factors and their correlation with slower cell proliferation in cells harboring dnIRE1, attesting to the fact that these genes expression is controlled by ER stress signaling and that ER stress is a necessary component of malignant tumor growth and cell survival. Moreover, the expression of some studied genes upon hypoxia is significantly depended on IRE1 signaling enzyme function. However, the detailed molecular mechanisms of this regulation are complex and warrants further studies.

\section{References}

Acosta-Alvear D, Zhou Y, Blais A, Tsikitis M, Lents NH, Arias C, Lennon CJ, Kluger Y, Dynlacht DD. XBP1 controls diverse cell type- and condition-specific transcriptional regulatory networks. Molecular Cell 27, 53-66, 2007.

Aragon T, van Anken E, Pincus D, Serafimova IM, Korennykh AV, Rubio CA, Walter P. Messenger RNA targeting to endoplasmic reticulum stress signalling sites. Nature 457, 736-740, 2009.

Auf G, Jabouille A, Guerit S, Pineau R, Delugin M, Bouchecareilh M, Favereaux A, Maitre M, Gaiser T, von Deimling A, Czabanka M, Vajkoczy P, Chevet E, Bikfalvi A, Moenner M. A shift from an angiogenic to invasive phenotype induced in malignant glioma by inhibition of the unfolded protein response sensor IRE1. Proc Natl Acad Sci USA 107, 15553-15558, 2010.

Auf G, Jabouille A, Delugin M, Guerit S, Pineau R, North S, Platonova N, Maitre M, Favereaux A, Vajkoczy P, Seno M, Bikfalvi A, Minchenko D, Minchenko O, Moenner M. High epiregulin expression in human U87 glioma cells relies on IRE1alpha and promotes autocrine growth through EGF receptor. BMC Cancer 13, 597, 2013.

Bochkov VN, Philippova M, Oskolkova O, Kadl A, Furnkranz A, Karabeg E, Breuss J, Minchenko OH, Mechtcheriakova D, Hohensinner P, Rychli K, Wojta J, Resink T, Binder BR, Leitinger N. Oxidized phospholipids stimulate angiogenesis via induction of VEGF, IL-8, COX-2 and ADAMTS-1 metalloprotease, implicating a novel role for lipid oxidation in progression and destabilization of atherosclerotic lesions. Circ Res 99, 900-908, 2006.

Bravo R, Parra V, Gatica D, Rodriguez AE, Torrealba N, Paredes F, Wang ZV, Zorzano A, Hill JA, Jaimovich E, Quest $\mathrm{AF}$, Lavandero $\mathrm{S}$. Endoplasmic reticulum and the unfolded protein response: dynamics and metabolic integration. Int Rev Cell Mol Biol 301, 215-290, 2013.

Cao SS, Kaufman RJ. Targeting endoplasmic reticulum stress in metabolic disease. Expert Opin Ther Targets 17, 437-448, 2013.

Chevet E, Hetz C, Samali A. Endoplasmic reticulum stress-activated cell reprogramming in oncogenesis. Cancer Discov 5, 586-597, 2015.

Chi MN, Guo ST, Wilmott JS, Guo XY, Yan XG, Wang CY, Liu XY, Jin L, Tseng HY, Liu T, Croft A, Hondermarck $\mathrm{H}$, Scolyer RA, Jiang CC, Zhang XD. INPP4B is upregulated and functions as an oncogenic driver through SGK3 in a subset of melanomas. Oncotarget 6, 39891-39907, 2015

Clarke HJ, Chambers JE, Liniker E, Marciniak SJ. Endoplasmic reticulum stress in malignancy. Cancer Cell 25, 563-573, 2014.

Dasgupta S, Putluri N, Long W, Zhang B, Wang J, Kaushik AK, Arnold JM, Bhowmik SK, Stashi E, Brennan CA, Rajapakshe K, Coarfa C, Mitsiades N, Ittmann MM, Chinnaiyan AM, Sreekumar A, O'Malley BW. Coactivator SRC-2-dependent metabolic reprogramming mediates prostate cancer survival and metastasis. J Clin Invest $125,1174-1188,2015$. 
de Guia RM, Herzig S. How do glucocorticoids regulate lipid metabolism? Adv Exp Med Biol 872, 127-144, 2015.

Dejeans N, Pluquet O, Lhomond S, Grise F, Bouchecareilh M, Juin A, Meynard-Cadars M, Bidaud-Meynard A, Gentil C, Moreau V, Saltel F, Chevet E. Autocrine control of glioma cells adhesion and migration through IRE1a-mediated cleavage of SPARC mRNA. J Cell Sci 125, 4278-4287, 2012.

Dejeans N, Barroso K, Fernandez-Zapico ME, Samali A, Chevet. Novel roles of the unfolded protein response in the control of tumor development and aggressiveness. Semin Cancer Biol 33, 67-73, 2015.

Drogat B, Auguste P, Nguyen DT, Bouchecareilh M, Pineau R, Nalbantoglu J, Kaufman RJ, Chevet E, Bikfalvi A, Moenner M. IRE1 signaling is essential for ischemia-induced vascular endothelial growth factor-A expression and contributes to angiogenesis and tumor growth in vivo. Cancer Res 67, 6700-6707, 2007.

Fenne IS, Helland T, Flageng MH, Dankel SN, Mellgren G, Sagen JV. Downregulation of steroid receptor coactivator- 2 modulates estrogen-responsive genes and stimulates proliferation of mcf-7 breast cancer cells. PLoS ONE 8, E70096, 2013.

Han J, Back SH, Hur J, Lin YH, Gildersleeve R, Shan J, Yuan CL, Krokowski D, Wang S, Hatzoglou M, Kilberg MS, Sartor MA, Kaufman RJ. ER-stress-induced transcriptional regulation increases protein synthesis leading to cell death. Nat Cell Biol 15, 481-490, 2013.

Han J, Kaufman RJ. Measurement of the unfolded protein response to investigate its role in adipogenesis and obesity. Methods Enzymol 538,135-150, 2014.

Hausmann S, Brandt E, Kochel C, Einsele H, Bargou RC, Seggewiss-Bernhardt R, Stuhmer T. Loss of serum and glucocorticoid-regulated kinase 3 (SGK3) does not affect proliferation and survival of multiple myeloma cell lines. PLoS One 10, e0122689, 2015.

Heiker JT, Kern M, Kosacka J, Flehmig G, Stumvoll M, Shang E, Lohmann T, Dressler M, Kovacs P, Bluher M, Kloting N. Nicotinamide nucleotide transhydrogenase mRNA expression is related to human obesity. Obesity (Silver Spring) 21, 529-534, 2013.

Huang Y, Zhou J, Huang Y, He J, Wang Y, Yang C, Liu D, Zhang L, He F. SARI, a novel target gene of glucocorticoid receptor, plays an important role in dexamethasone-mediated killing of B lymphoma cells. Cancer Lett 373, $57-66,2016$.

Khan SH, Ling J, Kumar R. TBP binding-induced folding of the glucocorticoid receptor AF1 domain facilitates its interaction with steroid receptor coactivator-1. PLoS ONE 6, E21939, 2011.

Kim IK, Kim BS, Koh CH, Seok JW, Park JS, Shin KS, Bae EA, Lee GE, Jeon H, Cho J, Jung Y, Han D, Kwon BS, Lee HY, Chung Y, Kang CY. Glucocorticoid-induced tumor necrosis factor receptor-related protein co-stimulation facilitates tumor regression by inducing IL-9-producing helper T cells. Nat Med 21, 1010-1017, 2015.

Langlais P, Yi Z, Finlayson J, Luo M, Mapes R, De Filippis E, Meyer C, Plummer E, Tongchinsub P, Mattern M, Mandarino LJ. Global IRS-1 phosphorylation analysis in insulin resistance. Diabetologia 54, 2878-2889, 2011.

Lee J, Ozcan U. Unfolded protein response signaling and metabolic diseases. J Biol Chem 289, 1203-1211, 2014.

Lenihan CR, Taylor CT. The impact of hypoxia on cell death pathways. Biochem Soc Trans 41, 657-663, 2013.

Li MD, Ruan HB, Singh JP, Zhao L, Zhao T, Azarhoush S, Wu J, Evans RM, Yang X. O-GlcNAc transferase is involved in glucocorticoid receptor-mediated transrepression. J Biol Chem 287, 12904-12912, 2012.

Liu ST, Lu GY, Hsu YJ, Chang LC, Ho CL, Huang SM. Dual roles for lysine 490 of promyelocytic leukemia protein in the transactivation of glucocorticoid receptor-interacting protein 1. Biochim Biophys Acta 1833, 1799-1810, 2013.

Liu H, Li C, Shen C, Yin F, Wang K, Liu Y, Zheng B, Zhang W, Hou X, Chen X, Wu J, Wang X, Zhong C, Zhang J, Shi H, Ai J, Zhao S. MiR-212-3p inhibits glioblastoma cell proliferation by targeting SGK3. J Neurooncol 122, 431-439, 2015.

Manie SN, Lebeau J, Chevet E. Cellular mechanisms of endoplasmic reticulum stress signaling in health and disease. 3. Orchestrating the unfolded protein response in oncogenesis: an update. Am J Physiol Cell Physiol 307, C901-C907, 2014.

Maurel M, Chevet E, Tavernier J, Gerlo S. Getting RIDD of RNA: IRE1 in cell fate regulation. Trends Biochem Sci 39, 245-254, 2014.

Maurel M, McGrath EP, Mnich K, Healy S, Chevet E, Samali A. Controlling the unfolded protein response-mediated life and death decisions in cancer. Semin Cancer Biol 33, 57-66, 2015.

Meimaridou E, Kowalczyk J, Guasti L, Hughes CR, Wagner F, Frommolt P, Nurnberg P, Mann NP, Banerjee R, Saka HN, Chapple JP, King PJ, Clark AJ, Metherell LA. Mutations in NNT encoding nicotinamide nucleotide transhydrogenase cause familial glucocorticoid deficiency. Nat Genet 44, 740-742, 2012.

Minchenko AG, Germanyuk YL. Effect of hydrocortisone on the expression of mitochondrial genes in the liver of normal and alloxan diabetic rats. Endocrinol Exper 18, 3-18, 1984. 
Minchenko AG. Effect of hydrocortisone on biosynthesis of mitochondrial and cytoplasmic RNA in liver of adrenalectomized rats. Endocrinol Exper 22, 75-86, 1988.

Minchenko AG, Tronjko ND. Subcellular distribution of 3H-hydrocortisone and its metabolites in the liver and kidneys of normal and alloxan diabetic rats. Endocrinol Exper 22, 19-28, 1988.

Minchenko OH, Kharkova AP, Bakalets TV, Kryvdiuk IV. Endoplasmic reticulum stress, its sensor and signaling systems and the role in regulation of gene expressions at malignant tumor growth and hypoxia. Ukr Biokhim Zh 85, 5-16, 2013a.

Minchenko DO, Kharkova AP, Hubenia OV, Minchenko OH. Insulin receptor, IRS1, IRS2, INSIG1, INSIG2, RRA D, and BAIAP2 gene expressions in glioma U87 cells with ERN1 loss of function: effect of hypoxia and glutamine or glucose deprivation. Endocr Regul 47, 15-26, $2013 \mathrm{~b}$.

Minchenko DO, Danilovskyi SV, Kryvdiuk IV, Bakalets TV, Lypova NM, Karbovskyi LL, Minchenko OH. Inhibition of ERN1 modifies the hypoxic regulation of the expression of TP53-related genes in U87 glioma cells. Endoplasm Reticul Stress Dis 1, 18-26, 2014.

Minchenko DO, Kharkova AP, Tsymbal DO, Karbovskyi LL, Minchenko OH. Expression of insulin-like growth factor binding protein genes and its hypoxic regulation in U87 glioma cells depends on ERN1 mediated signaling pathway of endoplasmic reticulum stress. Endocr Regul 49, 73-83, $2015 \mathrm{a}$.

Minchenko OH, Tsymbal DO, Moenner M, Minchenko DO, Kovalevska OV, Lypova NM. Inhibition of the endoribonuclease of ERN1 signaling enzyme affects the expression of proliferation-related genes in U87 glioma cells. Endoplasm Reticul Stress Dis 2, 18-29, 2015b.

Notsuda H, Sakurada A, Endo C, Okada Y, Horii A, Shima H, Kondo T. p190A RhoGAP is involved in EGFR pathways and promotes proliferation, invasion and migration in lung adenocarcinoma cells. Int J Oncol 43, 1569-1577, 2013.

Organ SL, Hai J, Radulovich N, Marshall CB, Leung L, Sasazuki T, Shirasawa S, Zhu CQ, Navab R, Ikura M, Tsao MS. p120RasGAP is a mediator of rho pathway activation and tumorigenicity in the DLD1 colorectal cancer cell line. PLoS One 9, e86103, 2014.

Pluquet O, Dejeans N, Bouchecareilh M, Lhomond S, Pineau R, Higa A, Delugin M, Combe C, Loriot S, Cubel G, Dugot-Senant N, Vital A, Loiseau H, Gosline SJ, Taouji S, Hallett M, Sarkaria JN, Anderson K, Wu W, Rodriguez FJ, Rosenbaum J, Saltel F, Fernandez-Zapico ME, Chevet E. Posttranscriptional regulation of PER1 underlies the oncogenic function of IREa. Cancer Res 73, 4732-4743, 2013.

Pluquet O, Dejeans N, Chevet E. Watching the clock: endoplasmic reticulum-mediated control of circadian rhythms in cancer. Ann Med 46, 233-243, 2014.

Psarra AM, Sekeris CE. Glucocorticoids induce mitochondrial gene transcription in HepG2 cells: role of the mitochondrial glucocorticoid receptor. Biochim Biophys Acta 1813, 1814-1821, 2011.

Pufall MA. Glucocorticoids and cancer. Adv Exp Med Biol 872, 315-333, 2015.

Qin J, Chen JX, Zhu Z, Teng JA. Genistein inhibits human colorectal cancer growth and suppresses miR-95, Akt and SGK1. Cell Physiol Biochem 35, 2069-2077, 2015.

Sheeran FL, Rydstrom J, Shakhparonov MI, Pestov NB, Pepe S. Diminished NADPH transhydrogenase activity and mitochondrial redox regulation in human failing myocardium. Biochim Biophys Acta 1797, 1138-1148, 2010.

Srivastava M, Leighton X, Starr J, Eidelman O, Pollard HB. Diverse effects of ANXA7 and p53 on LNCaP prostate cancer cells are associated with regulation of SGK1 transcription and phosphorylation of the SGK1 target FOXO3A. Biomed Res Int 2014, 193635, 2014.

Thomas AL, Coarfa C, Qian J, Wilkerson JJ, Rajapakshe K, Krett NL, Gunaratne PH, Rosen ST. Identification of potential glucocorticoid receptor therapeutic targets in multiple myeloma. Nucl Recept Signal 13, e006, 2015.

Vonlanthen J, Okoniewski MJ, Menigatti M, Cattaneo E, Pellegrini-Ochsner D, Haider R, Jiricny J, Staiano T, Buffoli F, Marra G. A comprehensive look at transcription factor gene expression changes in colorectal adenomas. BMC Cancer 14, 46, 2014.

Xiaobo Y, Qiang L, Xiong Q, Zheng R, Jianhua Z, Zhifeng L, Yijiang S, Zheng J. Serum and glucocorticoid kinase 1 promoted the growth and migration of non-small cell lung cancer cells. Gene 576, 339-346, 2016.

Zhao J, Xu H, He M, Wu Y. Glucocorticoid receptor DNA binding factor 1 expression and osteosarcoma prognosis. Tumour Biol 35, 12449-12458, 2014. 